\title{
Long term dynamics of the high luminosity Large Hadron Collider with crab cavities
}

\author{
J. Barranco García \\ EPFL, Lausanne CH-1015, Switzerland and CERN, Geneva 23 CH-1211, Switzerland
}

\author{
R. De Maria, A. Grudiev, and R. Tomás García \\ CERN, Geneva 23 CH-1211, Switzerland
}

\author{
R. B. Appleby and D. R. Brett \\ School of Physics and Astronomy, The University of Manchester, M13 9PL Manchester, United Kingdom \\ and Cockcroft Institute, Daresbury Laboratory, Warrington WA4 4AD, United Kingdom
}

(Received 23 June 2016; published 11 October 2016)

\begin{abstract}
The High Luminosity upgrade of the Large Hadron Collider (HL-LHC) aims to achieve an integrated luminosity of 200-300 $\mathrm{fb}^{-1}$ per year, including the contribution from the upgrade of the injector chain. For the HL-LHC the larger crossing angle together with a smaller beta function at the collision point would result in more than $70 \%$ luminosity loss due to the incomplete geometric overlap of colliding bunches. To recover head-on collisions at the high-luminosity particle-physics detectors ATLAS and CMS and benefit from the very low $\beta^{*}$ provided by the Achromatic Telescopic Squeezing (ATS) optics, a local crab cavity scheme provides transverse kicks to the proton bunches. The tight space constraints at the location of these cavities leads to designs which are axially non-symmetric, giving rise to high order multipoles components of the main deflecting mode and, since these kicks are harmonic in time, we expand them in a series of multipoles in a similar fashion as is done for static field magnets. In this work we calculate, for the first time, the higher order multipoles and their impact on beam dynamics for three different crab cavity prototypes. Different approaches to calculate the multipoles are presented. Furthermore, we perform the first calculation of their impact on the long term stability of the machine using the concept of dynamic aperture.
\end{abstract}

DOI: 10.1103/PhysRevAccelBeams.19.101003

\section{INTRODUCTION}

The Large Hadron Collider (LHC) will undergo a major upgrade around 2026 to increase its discovery potential. The goal is to provide the experiments with $3000 \mathrm{fb}^{-1}$ over 12 years, which implies between $250-300 \mathrm{fb}^{-1}$ per year. The target peak luminosity in this new scenario for the two high-luminosity experiments ATLAS and CMS is $7.4 \times$ $10^{34} \mathrm{~cm}^{-2} \mathrm{~s}^{-1}$ (leveled during a fill to $5 \times 10^{34} \mathrm{~cm}^{-2} \mathrm{~s}^{-1}$ ) $[1,2]$. For this reason this upgraded machine is referred to as High Luminosity LHC (HL-LHC). The main enhancements foreseen in this scenario include new high-field and large-aperture inner triplet quadrupoles used to squeeze the beam and bring it into collision, use of crab cavities to recover head on collisions at interaction point 1 (IP1) and IP5 and various improvements in the experiments to sustain higher luminosity levels. A detailed description of all new equipments and interventions foreseen for the HL-LHC can

Published by the American Physical Society under the terms of the Creative Commons Attribution 3.0 License. Further distribution of this work must maintain attribution to the author(s) and the published article's title, journal citation, and DOI. be found in [2]. The HL-LHC is also foreseen to feature new collision optics, namely achromatic telescopic squeezing (ATS) [3] which will provide very low values of $\beta^{*}$ (the betatron function at the IP) and at the same time ensure a good correction of the chromatic aberrations. In order to fully benefit from the lower $\beta^{*}$, crab cavities are essential to counteract the geometric luminosity reduction due to a large crossing angle and to limit the event pile-up density. The geometric reduction factor $(R)$ depends on the beam size at the IP and the collision crossing angle as (neglecting the hour glass effect),

$$
R(\theta)=\frac{1}{\sqrt{1+\left(\frac{\sigma_{z}}{\sigma_{x, y}^{*}} \frac{\theta}{2}\right)^{2}}},
$$

with $\sigma_{x, y}^{*}$ the traverse beam size at the IP, $\sigma_{z}$ the longitudinal beam size and $\theta / 2$ half of the crossing angle. In essence the increase in luminosity due to the small $\sigma_{x, y}^{*}$ is lost given the large crossing angle needed to overcome long range beambeam interaction. Assuming HL-LHC parameters [1], the expected geometric luminosity reduction is around $70 \%$ (Table I). A crab crossing scheme would recover head on collisions, overcoming this loss of luminosity to fully benefit from the new proposed optics. 
TABLE I. HL-LHC parameters for the two bunch spacing options 25 and $50 \mathrm{~ns}$ in comparison with LHC nominal [1]. The geometrical reduction factor does not include the hour glass effect.

\begin{tabular}{lccc}
\hline \hline & \multirow{2}{*}{ LHC } & \multicolumn{2}{c}{ HL-LHC } \\
Parameter & Nominal & $25 \mathrm{~ns}$ & $50 \mathrm{~ns}$ \\
\hline $\mathrm{N}\left[10^{11} \mathrm{ppb}\right]$ & 1.15 & 2.2 & 3.5 \\
$\mathrm{n}_{b}$ & 2808 & 2808 & 1404 \\
Crossing Angle $[\mu \mathrm{rad}]$ & 300 & 590 & 590 \\
$\beta^{*}[\mathrm{~m}]$ & 0.55 & 0.15 & 0.15 \\
$\epsilon_{n}[\mu \mathrm{m}]$ & 3.75 & 2.5 & 3.0 \\
$\sigma_{z}[\mathrm{~cm}]$ & 7.5 & 7.5 & 7.5 \\
Geom. Reduction & 0.83 & 0.305 & 0.331 \\
Peak lumi $\left[10^{34} \mathrm{~cm}^{-2} \mathrm{~s}^{-1}\right]$ & 1.0 & 7.4 & 8.5 \\
Virtual lumi[1034 $\left.\mathrm{cm}^{-2} \mathrm{~s}^{-1}\right]$ & 1.2 & 24 & 26 \\
\hline \hline
\end{tabular}

The crab cavities (CCs) are rf deflecting cavities operated with a $90^{\circ}$ phase shift, giving a $z$-dependent transverse kick to the bunch. The crabbing action is defined so the center of each bunch remains undeflected, whereas the head is kicked to one side and the tail to the other. The total effect is a tilt of half the crossing angle $(\theta / 2)$ with respect to the uncrabbed motion at the IP [4,5]. The $z$-dependence translates into different closed orbits for different longitudinal positions, which will experience different feed down errors when passing off-center in the interaction region (IR) elements. This is the basic principle used in CLIC to enhance luminosity in a traveling waist regime [6].

In this paper, the HLLHCV1.1 version [7] of the HL-LHC optics characterized by a $\beta_{x, y}^{*}=15 \mathrm{~cm}$ (round optics), quadrupoles in the focusing triplets with $140 \mathrm{~T} / \mathrm{m}$ field gradient and half crossing angle $\theta / 2=295 \mu \mathrm{rad}$ at IP1 and 5 (defined as the half of the angle formed by the closed orbit of the two counterrotating beams). The bunch spacing is $25 \mathrm{~ns}$. The crab cavities will be installed in a location that provides large $\beta$ functions and a phase advance to the IP of $\pi / 2$ in order to minimize the required voltage in the cavity. Furthermore, in order to close the crab orbit bump a second set of CCs after the IP at $\pi / 2$ phase advance is needed. This is referred to as the local scheme since the orbit bump is closed locally around the IP. To keep the cavity surface fields below the limits of $60 \mathrm{MV} / \mathrm{m}$ and $100 \mathrm{mT}$ [8] and to minimize the impact of failure scenarios, 3 modules per beam and per IP side are considered.

Physically, the crab cavities should fit in between the two beam lines near the separation dipole D2 [9]. This space constraint, plus the $400 \mathrm{MHz}$ rf frequency requirements, forces the design to be extremely compact, breaking the axial symmetry of the cavity, and hence giving rise to time varying higher order multipoles. These multipoles vary for the three crab cavities prototypes (ridged waveguide, four rod resonator, and quarter-wave resonator) as each of them exploit different compact geometries (Fig. 1) to make a $400 \mathrm{MHz}$ deflecting cavity. All cavities are oriented so the particles move parallel to the $z$ axis and the dipolar kick is in the $x$ direction.

The crab cavity beam dynamics have been studied in [14-16]. In this paper differing methods to compute rf multipole content of the CCs are presented, characterizing the dynamics in the cavity using multipoles, analogous to the standard treatment of magnets. We make the first complete calculations of the multipole content of all three crab cavity designs for the LHC and make the first assessment of the expected effect on the single particle long term stability in a proton machine. The tolerance of general crab cavity designs to multipole magnitude is also discussed and optical aberrations calculated. The result is a comprehensive understanding of the multipole content of symmetric and nonsymmetric crab cavities and the first assessment of the resulting dynamics in a proton machine.

The layout of this paper is as follows. In Sec. II we describe the methodology used to calculate the multipolar coefficients and present the coefficients for the three designs proposed for the LHC. Here a thin lens model
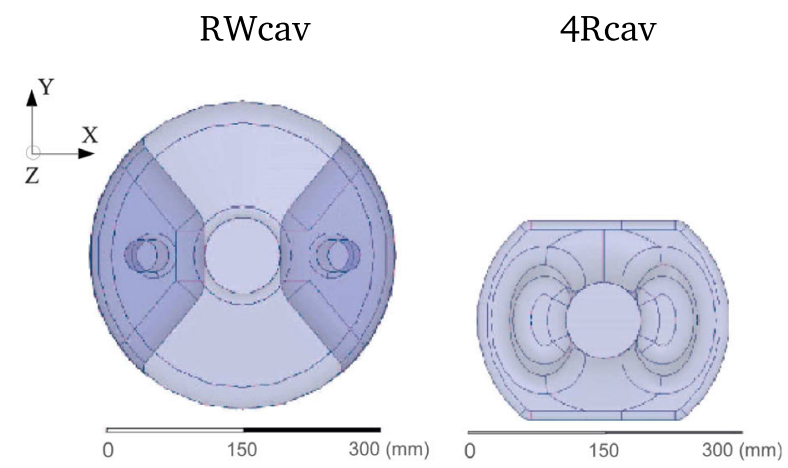

QWcav 2011

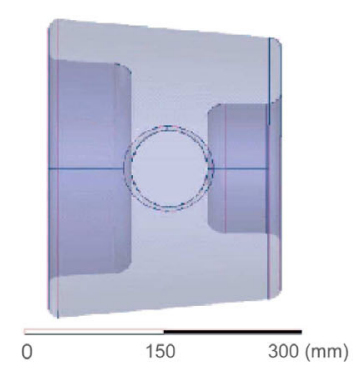

QWcav 2012

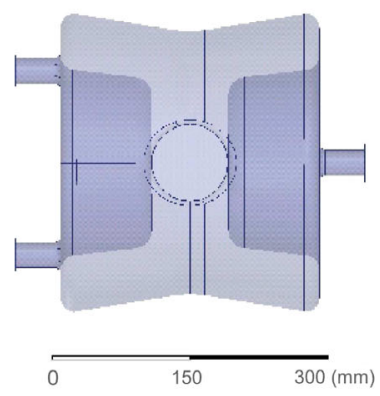

FIG. 1. Geometry of the four HL-LHC CC prototypes under design: ridged waveguide cavity (RWcav) [10]; four rod resonator cavity (4Rcav) [11]; quarter-wave resonator cavity 2011 (QWcav 2011) [12] and double quarter-wave resonator (QWcav 2012) [13]. 
approximation will be used but a more realistic description of the crab cavities using Taylor Maps can be found in [17]. In Sec. III these multipoles are used to study the long term beam dynamics of the LHC in the presence of crab cavities, in order to assess, with a realistic crab cavity model. the impact on the machine's dynamic aperture. The optical aberrations in terms of multipole strength are computed to assess the strength of the dependence and allow assessments of aberrations for cavities with general multipole content. Finally we draw our conclusions in Sec. IV.

\section{THIN LENS RF MULTIPOLE MODEL}

The strong nonaxially symmetric shape of the compact cavities presented in Fig. 1 leads to a transverse dependency of the transverse kicks applied by the operating dipole mode. This effect can be described in a similar way as is done in the analysis of static magnetic fields by means of an expansion of the electromagnetic (EM) fields of the cavities and associated transverse and longitudinal kicks in terms of azimuthal multipoles. In order to do so, some approximations are assumed. First the crab cavities are represented by thin lens elements, i.e. the 3D field region is replaced by two drifts around a thin layer with infinite fields. The particle trajectory is not affected by the field while traversing the cavity and a kick is only transmitted to it at the center of the cavity. Furthermore the paraxial approximation is assumed. Assuming harmonic time dependence of complex EM fields, the particle velocity is equal to the speed of light and the fields vanish at the boundary, the integrated vector potential can be reduced to the following form,

$$
\begin{aligned}
\int_{0}^{L} A_{s}(\rho, \phi, s) \exp \left(\frac{\mathrm{i} \omega s}{c}\right) d s \\
=\sum_{n=1}^{N} \frac{1}{n} \rho^{n}\left[b_{n} \cos (n \phi)+a_{n} \sin (n \phi)\right]
\end{aligned}
$$

where $\omega$ is rf frequency, $c$ is the speed of light, and $b_{n}$ and $a_{n}$ are the normal and skew multipolar expansion coefficients of the complex EM fields, analogous to the description used for magnets but complex instead of real, and $N$ is the order of truncation of the azimuthal decomposition [18]. This integrated vector potential is expressed in a thin-lens form, with no $s$ dependence, and $a_{n}$ and $b_{n}$ are the integrated values over the length of the cavity. From the vector potential in this form the momentum change applied to a particle can be calculated in complex form as

$$
\Delta p_{\perp}(\rho, \phi)=q \int_{0}^{L} \nabla_{\perp} A_{s} \exp \left(\frac{\mathrm{i} \omega s}{c}\right) d s
$$

$$
\begin{aligned}
& =q \int_{0}^{L}\left(\begin{array}{c}
\frac{\partial A_{s}}{\partial \rho} \hat{\rho} \\
\frac{1}{\rho} \frac{\partial A_{s}}{\partial \phi} \hat{\phi}
\end{array}\right) \exp \left(\frac{\mathrm{i} \omega s}{c}\right) d s \\
& =q \sum_{n=1}^{N} \rho^{n-1}\left(\begin{array}{c}
\left(b_{n} \cos (n \phi)+a_{n} \sin (n \phi)\right) \hat{\rho} \\
\left(a_{n} \cos (n \phi)-b_{n} \sin (n \phi)\right) \hat{\phi}
\end{array}\right) .
\end{aligned}
$$

The momentum change experienced by a particle traversing the cavity parallel to its axis can then be expressed as

$$
\Re\left\{\Delta p_{\perp}(\rho, \phi) \exp \left[\mathrm{i}\left(\frac{\omega}{c} z+\Phi\right)\right]\right\},
$$

where $z$ is the longitudinal position of a particle in the bunch and $\Phi$ is the rf phase. The rf phase is usually chosen for convenience to be either a deflecting phase, in which case the kick $\Delta p_{\perp}$ due to the main multipole $n=1$ is purely real and particle at $z=0$ experiences maximum deflection, or a crabbing phase, in which case the kick due to the main multipole $n=1$ is purely imaginary and the particle at $z=0$ sees no kick. In this section we describe the methods used to calculate the multipole coefficients of the cavities in section A, and present the multipole coefficients themselves in section B.

\section{A. Multipolar decomposition methods}

EM field maps have been calculated for the three cavity geometries presented in Fig. 1 using frequency domain finite element code HFSS [19]. A precise Fourier decomposition of the multipole components requires a regular meshing upon the surface of a cylinder. This avoids the introduction of non-Maxwellian interpolation which was found to lead to significant variation in the multipole results. To enable this, a large amount of computation is required to determine the field values on this cylindrical surface. A mesh is produced at $10 \mathrm{~mm}$ and $20 \mathrm{~mm}$ with 16 uniform azimuthally separated mesh points, at regular longitudinal positions. The $E$ and $B$ fields are then extracted and three different decomposition methods applied in order to calculate the multipolar expansion coefficients. These three methods are described in the following sections.

\section{Lorentz force}

Starting from the general form of the Lorenz force law (LF) the transverse component of the force experienced by a particle moving along $z$-axis is given by,

$$
F_{\perp}(\rho, \phi, s)=q\left[E_{\perp}+(\vec{v} \times \vec{B})_{\perp}\right] \exp \left(\mathrm{i} \frac{\omega s}{v}\right),
$$


where $\rho, \phi$ and $s$ are cylindrical coordinates, $q$ is the particle charge and $\vec{E}$ and $\vec{B}$ are the complex electric and magnetic fields respectively. The momentum kick can be found from the force by integrating over the length of the cavity $L$,

$$
\Delta p_{\perp}(\rho, \phi)=\frac{1}{c} \int_{0}^{L} F_{\perp} d s,
$$

where $v=c$ is assumed. Combining this kick with Eqs. (3) we find an expression for the radial kick $\Delta p_{\rho}$,

$\frac{1}{c} \int_{0}^{L} F_{\rho} d s=q \sum_{n=1}^{N} \rho^{n-1}\left[b_{n} \cos (n \phi)+a_{n} \sin (n \phi)\right]$.

Hence the multipolar expansion coefficients are found by a Fourier transformation of the radial force $F_{\rho}$,

$a_{n}=\frac{1}{q c} \frac{1}{\pi} \int_{-\pi}^{\pi} \frac{1}{\rho^{n-1}} \sin (n \phi) \int_{0}^{L} F_{\rho}(\rho, \phi, s) d s d \phi$

$b_{n}=\frac{1}{q c} \frac{1}{\pi} \int_{-\pi}^{\pi} \frac{1}{\rho^{n-1}} \cos (n \phi) \int_{0}^{L} F_{\rho}(\rho, \phi, s) d s d \phi$.

If necessary the multipolar expansion coefficients can be calculated from a Fourier transformation of azimuthal kick $\Delta p_{\phi}$ or the transverse kick components in Cartesian coordinates, $\Delta p_{x}$ or $\Delta p_{y}$, using expressions derived in a similar way to the above equations.

\section{Panofsky-Wenzel}

The Panofsky-Wenzel (PW) theorem [20] is a useful relationship between transverse and longitudinal kicks allowing the multipolar expansion coefficients to be calculated from the longitudinal electric field. Assuming the ultrarelativistic case $(v \rightarrow c)$ and no fringe fields beyond the edges of the integrable region $[0, L]$, the momentum change can be expressed in terms of $E_{s}$ only,

$\Delta p_{\perp}(\rho, \phi)=q \frac{\mathrm{i}}{\omega} \int_{0}^{L} \nabla_{\perp} E_{s}(\rho, \phi, s) \exp \left(\mathrm{i} \frac{\omega s}{c}\right) d s$.

From this equation, Eq. (2) and Eq. (3), the following relation can be found.

$$
\begin{aligned}
& \frac{\mathrm{i}}{\omega} \int_{0}^{L} E_{s}(\rho, \phi, s) \exp \left(\mathrm{i} \frac{\omega s}{c}\right) d s \\
& \quad=\sum_{n=1}^{N} \frac{1}{n} \rho^{n}\left[b_{n} \cos (n \phi)+a_{n} \sin (n \phi)\right] .
\end{aligned}
$$

Hence the multipolar expansion coefficients are found by a Fourier transformation of the electric field,

$$
\begin{aligned}
& a_{n}=\frac{\mathrm{i} n}{\omega} \frac{1}{\pi} \int_{-\pi}^{\pi} \frac{1}{\rho^{n}} \sin (n \phi) \int_{0}^{L} e^{\mathrm{i} \frac{\omega}{c} s} E_{s}(\rho, \phi, s) d s d \phi \\
& b_{n}=\frac{\mathrm{i} n}{\omega} \frac{1}{\pi} \int_{-\pi}^{\pi} \frac{1}{\rho^{n}} \cos (n \phi) \int_{0}^{L} e^{\mathrm{i} \frac{\mathrm{\omega} s}{c}} E_{s}(\rho, \phi, s) d s d \phi,
\end{aligned}
$$

using only $E_{z}$.

\section{Helmholtz decomposition}

An equivalent approach to computing the multipoles can be used by noting the fields obey the Helmholtz equation and exploiting the Fourier decomposition method developed in [21]. This method was applied to trajectories in the LHC crab cavities in [22] by integrating through the $s$ dependent fields, and in this section we use this formulation as an alternative way (but entirely equivalent to the PW method) to compute the multipoles. A complete study of crab cavity dynamics using the Helmholtz decomposition is performed in [17].

The Helmholtz method proceeds by taking the solution for $E_{s}(\mathbf{r})$ in the form,

$$
\begin{aligned}
E_{s}(\mathbf{r})= & \int_{-\infty}^{\infty} \frac{d k}{\sqrt{2 \pi}} e^{\mathrm{i} k s}\left(\tilde{e}_{0}(k) R_{0}(k, \rho)\right. \\
& +\sum_{n=1}^{\infty}\left[\tilde{e}_{n}(k) R_{n}(k, \rho) \cos (n \phi)\right. \\
& \left.\left.+\tilde{f}_{n}(k) R_{n}(k, \rho) \sin (n \phi)\right]\right)
\end{aligned}
$$

where $R_{n}$ is defined,

$$
R_{n}(k, \rho)= \begin{cases}J_{n}\left(\kappa_{l}(k) \rho\right) & \text { If } \operatorname{sgn}\left(k^{2}-k_{l}^{2}\right)<0, \\ I_{n}\left(\kappa_{l}(k) \rho\right) & \text { Otherwise. }\end{cases}
$$

In this form $k_{l}$ is the wave vector for the given mode in the cavity and $n$ is the order of the Bessel function and multipolar expansion term. The function $\kappa_{l}$ is defined by the relation of $k_{l}$ to $k$,

$$
\kappa_{l}^{2}(k)=\left|k^{2}-k_{l}^{2}\right| .
$$

The functions $\tilde{e}(k)$ and $\tilde{f}(k)$ are calculated from the field data, which are meshed over $(\phi, z)$ at $\rho=R$. From this data, Fourier transforms are carried out to obtain the Fourier decomposition of the harmonic modes of the fields, defining $\tilde{e}_{n}(k)$ for $n \in\{0,1,2, \ldots\}$ as

$$
\begin{aligned}
\tilde{e}_{n}(k)= & \frac{1}{R_{n}(k, R)} \int_{-\infty}^{\infty} \frac{d s}{\sqrt{2 \pi}} e^{-\mathrm{i} k s} \\
& \times \int_{-\infty}^{\infty} \frac{d \phi}{\pi} \cos (n \phi) E_{s}(R, \phi, s) .
\end{aligned}
$$

Similarly $\tilde{f}_{n}(k)$ is defined for $n \in\{1,2, \ldots\}$ as 
TABLE II. Values of the multipolar rf multipoles for the crab cavity prototypes at nominal deflecting voltage: $\mathrm{V}_{c c}=10 \mathrm{MV}$ in units of $\mathrm{mTm} / \mathrm{m}^{n-1}$.

\begin{tabular}{|c|c|c|c|c|c|c|}
\hline & & \multicolumn{2}{|c|}{ Lorentz method } & \multicolumn{2}{|c|}{ Panofsky-Wenzel } & \multirow{2}{*}{$\begin{array}{l}\text { Helmholtz decom } \\
\text { position@ @ } 20 \mathrm{~mm}\end{array}$} \\
\hline & & @ 10 mm & @ 20 mm & @ 10 mm & @ $20 \mathrm{~mm}$ & \\
\hline 4RCAV & $b_{2}$ & -0.06 & -0.05 & -0.06 & -0.06 & -0.10 \\
\hline \multirow[t]{2}{*}{2012} & $b_{3}$ & 1159 & 1159 & 1161 & 1161 & 1156 \\
\hline & $b_{4}$ & -4 & 100 & 65 & 27 & 57 \\
\hline RWCAV & $b_{2}$ & 0.01 & 0.00 & 0.00 & 0.01 & 0.02 \\
\hline \multirow[t]{2}{*}{2012} & $b_{3}$ & 4511 & 4511 & 4495 & 4495 & 4518 \\
\hline & $b_{4}$ & -4 & -7 & -21 & 7 & 10 \\
\hline QWCAV & $b_{2}$ & 111.42 & 111.40 & 111.43 & 111.48 & 113.06 \\
\hline \multirow[t]{2}{*}{2011} & $b_{3}$ & 1266 & 1267 & 1257 & 1260 & 1279 \\
\hline & $b_{4}$ & 1776 & 1776 & 1401 & 1836 & 2102 \\
\hline QWCAV & $b_{2}$ & 0.29 & 0.29 & 0.29 & 0.29 & 0.24 \\
\hline \multirow[t]{2}{*}{2012} & $b_{3}$ & 1074 & 1073 & 1078 & 1078 & 1073 \\
\hline & $b_{4}$ & 50 & 67 & 6 & 64 & 22 \\
\hline
\end{tabular}

$$
\begin{aligned}
\tilde{f}_{n}(k)= & \frac{1}{R_{n}(k, R)} \int_{-\infty}^{\infty} \frac{d s}{\sqrt{2 \pi}} e^{-\mathrm{i} k s} \\
& \times \int_{-\infty}^{\infty} \frac{d \phi}{\pi} \sin (n \phi) E_{s}(R, \phi, s) .
\end{aligned}
$$

Given $\tilde{e}$ and $\tilde{f}$, the multipolar coefficients $a_{n}$ and $b_{n}$ can be obtained using the expressions

$$
\begin{aligned}
& a_{n}=\frac{\mathrm{i} n}{\omega} \int_{0}^{L} d s e^{\mathrm{i} k_{l} s} \int_{-\infty}^{\infty} \frac{d k}{\sqrt{2 \pi}} e^{\mathrm{i} k z} \tilde{f}_{n}(k) \frac{\left|k^{2}-k_{l}^{2}\right|^{\frac{n}{2}}}{2^{n} n !} \\
& b_{n}=\frac{\mathrm{i} n}{\omega} \int_{0}^{L} d s e^{\mathrm{i} k_{l} s} \int_{-\infty}^{\infty} \frac{d k}{\sqrt{2 \pi}} e^{\mathrm{i} k z} \tilde{e}_{n}(k) \frac{\left|k^{2}-k_{l}^{2}\right|^{\frac{n}{2}}}{2^{n} n !} .
\end{aligned}
$$

Note these coefficients obtained are equivalent to those obtained using the PW method but through the functions $\tilde{e}(k)$ and $\tilde{f}(k)$.

\section{B. Multipolar coefficients of prototypes}

The computed multipole coefficients are presented in Table II using the three methods presented in Sec. II. These dipolar cavities present the same behavior of the magnets in terms of allowed multipoles, and so the first allowed multipole is sextupole for symmetric cavities. The imaginary part, not shown, in all cases is zero within the numerical accuracy of the calculation. It means that all

TABLE III. Values of the magnetic multipoles in the LHC superconducting separation dipoles (D2).

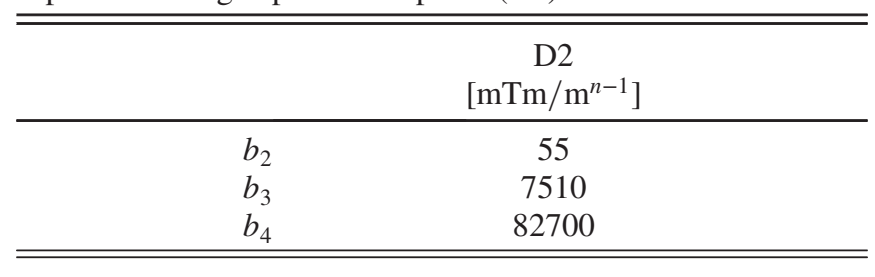

higher order multipoles $(n>1)$ are in phase with the main dipole component $(n=1)$ and there is no transverse kick acting on a particle at the crabbing phase. For upright cavities as shown in Fig. 1 all skew multipoles are zero and, in addition, if they present biaxial transverse symmetry, only odd multipoles coefficients are exhibited. However due to systematic errors in the calculation of the multipoles some small even multipoles are predicted. The QWCAV 2011 prototype has only one transverse symmetry and hence additionally contains even multipoles. Table II also includes the multipoles for the QWCAV 2012, which was redesigned to reduce the $b_{2}$ multipole component. A comparison of the dynamics of the two designs will be performed in this paper.

All three methods show very good agreement between them, with some differences in the prediction for $b_{4}$. For example the LF method presents errors introduced by the numerical methods used to find the magnetic field from the electric field in the eigenmode solver. On the other hand the PW and Helmholtz methods only require electric field data. For the dynamic aperture results in this paper the Lorentz method at $10 \mathrm{~mm}$ is used.

In order to give a scale to the rf multipolar strength for LHC CC prototypes, they can be compared to the field quality of the D2 separation dipoles that are currently installed in the LHC ring in IR1 and IR2 [23]. These multipoles are shown in Table III. It is interesting to note that the rf multipoles are lower but of the same order of magnitude as the magnetic ones. This means that a careful analysis of the impact of the CC field quality is mandatory and its impact on the long-term beam dynamics should be addressed.

\section{IMPACT ON THE BEAM DYNAMICS}

In this section we evaluate the impact of the multipole content of the crab cavities, both in general terms by assessing the impact of generic multipoles on properties 
such as tune and chromaticity of circular machines, by analytically computing the resulting optics aberrations expected depending on the order of the multipole, and in terms of the explicit dynamic aperture impact at the LHC. These two approaches evaluate the expected effect of the CCs on the beam dynamics of the HL-LHC, as well as multipole tolerances. For the assessment of the LHC, a full dynamic aperture scan is performed to evaluate the impact of the crab cavity multipoles on the long-term stability of the beam.

The LHC has four interaction points but only two of them (IP1 and 5) will likely feature a local crab crossing scheme. The crossing plane for each one is still under discussion; an alternating crossing scheme $\left(\mathrm{V}_{\mathrm{IP1}}-\mathrm{H}_{\mathrm{IP} 5}\right)$ is the most favorable from the beam-beam effects point of view [24]. The $\mathrm{H}_{\mathrm{IP1}}-\mathrm{H}_{\mathrm{IP} 5}$ case is included as well for comparison. In this work the round beam optics $\left(\beta_{x}^{*}=\beta_{y}^{*}\right)$ is used. Note that beam-beam effects are not part of the simulations in this study but some results can be found in [25].

In the case of symmetric upright designs, i.e. horizontal crossing scheme, all multipolar components are normal $\left\{b_{2}, b_{3}, b_{4}\right\}$ (Table II). For the case of vertical crossing angle, the cavity is rotated by $90^{\circ}$ so the multipolar components become $\left\{-b_{2}, 0, b_{4}\right\}$ and skew $\left\{0, b_{3}, 0\right\}$. The real cavities, however, will exhibit deviations from these ideal symmetries so any combinations of multipoles could arise. In a $\mathrm{H}_{\mathrm{IP} 1}-\mathrm{H}_{\mathrm{IP} 5}$ scheme all multipolar components will add up. In particular the quadrupolar component $b_{2}$ will add up producing a non-negligible tune shift, as seen in the following section.

In the case of $\mathrm{V}_{\mathrm{IP1}}-\mathrm{H}_{\mathrm{IP} 5}$ the quadrupolar component cancels out between the IPs, which mitigates what otherwise would be a significant concern from a long term stability point of view. In case of nonsymmetric cavity designs, where $b_{n} \neq 0$ with $n$ even, the noncancellation of $b_{2}$ produces a non-negligible tuneshift in a $\mathrm{H}_{\mathrm{IP1}}-\mathrm{H}_{\mathrm{IP} 5}$ scenario that might compromise the long term stability of the machine. This tuneshift is modulated by the synchrotron period so can only be corrected with other rf multipoles.

In both simulations and analytical estimations the $\mathrm{H}_{\mathrm{IP1}}-\mathrm{H}_{\mathrm{IP5}}$ scenario is used since it is the worst case scenario.

\section{A. Analytical estimations}

The presence of crab cavity nonlinearities will introduce different type of beam dynamics perturbations into the lattice's beam dynamics, and the rf multipoles differ from the magnetic multipoles by the time dependency. In this work we evaluate a worst case scenario by taking the modulus of the rf kick. Analytically the aberrations for normal and skew multipoles are [26], (i) Linear tune shift,

$$
\Delta Q_{x, y}=\frac{1}{4 \pi} \beta_{x, y} \frac{b_{2}}{B \rho} .
$$

(ii) The minimum tune split from the linear coupling,
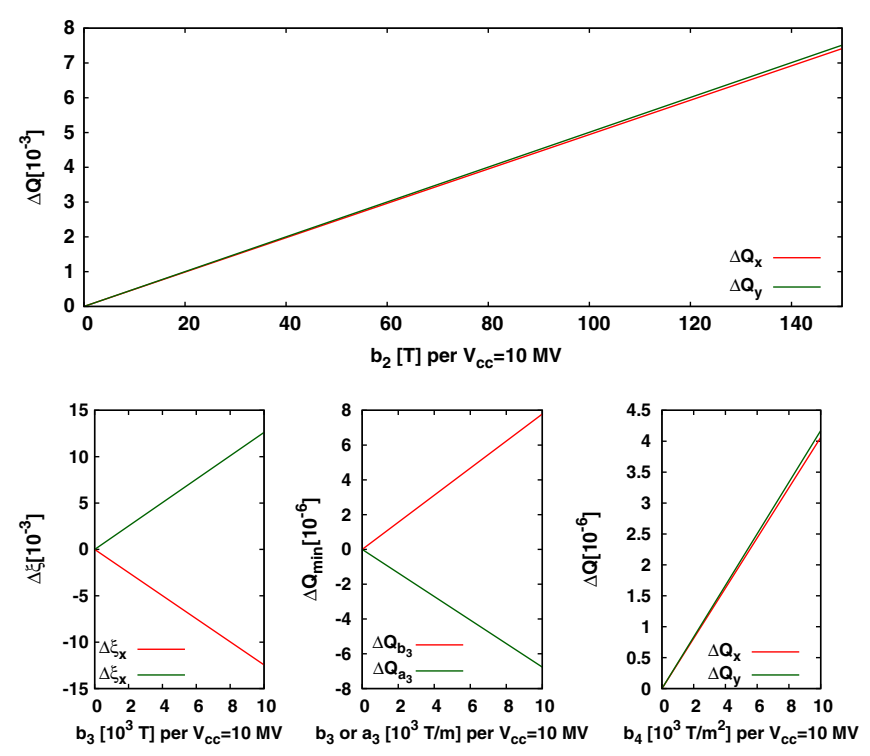

FIG. 2. Optics aberrations for the different multipole orders, as a function of coefficient magnitude.

$$
\Delta Q_{\min }=\frac{1}{4 \pi} \sqrt{\beta_{x} \beta_{y}} \frac{a_{2}}{B \rho} .
$$

(iii) Chromaticity shift,

$$
\Delta \xi_{x, y}=\frac{ \pm 1}{4 \pi} D_{x} \beta_{x, y} \frac{2 b_{3}}{B \rho} .
$$

(iv) Chromatic coupling,

$$
\frac{\partial Q_{\min }}{\partial \delta}=\frac{1}{2 \pi} \sqrt{\beta_{x} \beta_{y}} D_{y, x} \frac{2(b, a)_{3}}{B \rho} .
$$

(v) Amplitude detuning, generating a tune shift at $3 \sigma$ (the coupling term is not considered here)

$$
\Delta Q_{x, y}=\frac{3}{8 \pi} \beta_{x, y}^{2} \frac{b_{4}}{B \rho} 9 J_{x, y},
$$

where $\beta_{x, y}$ and $D_{x, y}$ are the betatronic and dispersion function at the location of the multipole and $B \rho, \delta$ and $J_{x, y}$ the magnetic rigidity of the beam, the relative energy spread and the action. These options aberrations have been evaluated for LHC parameters in Fig. 2, which shows the optics aberration as a function of normal multipole coefficient $b_{n}$. The top plot shows the tuneshift as a function of $b_{2}$, showing a potentially large tuneshift for even moderate values of $b_{2}$, which could cause beam dynamical issues in the machines containing these cavities. The figure hence shows the importance of suppressing $b_{2}$ from symmetric cavity design. For other optical aberrations linked to $b_{3}$ or $b_{4}$, the dependence of the aberrations on the coefficient is weak, and the magnitude is generally small for all aberrations. A further implication of this study is the alignment of the cavities 
has to be carefully done to avoid a large $b_{2}$ resulting from feeddown in a presence of $b_{3}$.

\section{B. Dynamic aperture of the LHC with crab cavities}

The long term stability of the HL-LHC is evaluated in this work for the first time in the presence of crab cavities, modeled with time dependent multipoles. A campaign of dynamic aperture calculations using the SixTrack SixDesk Environment [27] has been performed with an updated version of SixTrack including rf multipoles up to the 4th order (See Appendix for the explicit form of the kicks in the cavity up to 4th order). The dynamic aperture (DA) estimates the transverse chaotic boundary below which the particles have a stable motion in the long term. The DA results are expressed in terms of the transverse rms beam size $\sigma$, with $\epsilon_{n_{x, y}}$ being the HL-LHC normalized emittance at $1 \sigma$ in both planes and equal to $2.5 \mu \mathrm{rad}$. The calculations use a sampling steps of 2/30 $\sigma$ in amplitude and 1.8 degrees step in angle. The relative momentum deviation is chosen traditionally $\delta p / p=0.0027$ to maximize the chromatic perturbations. In addition to the different particles amplitude each particle is initialized with a different phase space angle defined, defined as,

$$
\phi=\arctan \sqrt{\frac{\epsilon_{y}}{\epsilon_{x}}} .
$$

The particle motion will sample different resonances depending on the ratio between horizontal and vertical oscillation amplitudes and, for this reason, 59 phase space angles equally spaced are evaluated per amplitude step. The particles are tracked for a total of $10^{5}$ turns and the minimum DA ( $\mathrm{DA}_{\min }$ - dashed lines) is used to characterize the different scenarios. In the results to follow, the mean DA of all seeds ( $\mathrm{DA}_{\text {mean }}$-solid lines) is plotted as well for reference. Magnetic multipole errors in the inner triplet (IT) are taken into account (version 6.5 [28]), as well as the errors in the magnets of the arcs. In all cases appropriate beam corrections are applied. The dynamic aperture of the machine is defined as the minimum of all dynamic apertures over sixty statistical realizations of the error model.

In all simulations we considered the worst case scenario of $\mathrm{H}_{\mathrm{IP1}}-\mathrm{H}_{\mathrm{IP} 5}$ where all the multipoles contributions (including $b_{2}$ ) will add up. To illustrate this, the top plot of Fig. 3 shows the average and minimum DA results for a range of values of $b_{2}$ and $a_{2}$. For a nonsymmetric cavity (the quarter wave 2011 design) in the YZ plane, the large tuneshift produced by the $b_{2}$ together with the fact that this is not compensated by the other cavity as in a $\mathrm{V}_{\mathrm{IP} 1}-\mathrm{H}_{\mathrm{IP} 5}$ scenario led to a minimum DA of about $7 \sigma$. The 2012 redesign of the cavity to a symmetric configuration to suppress the quadrupolar multipole removes this damaging beam dynamical effect. Figures 3 middle and bottom, shows the minimum and average DA as a function of multipole coefficient and shows these sextupolar and octupolar multipolar
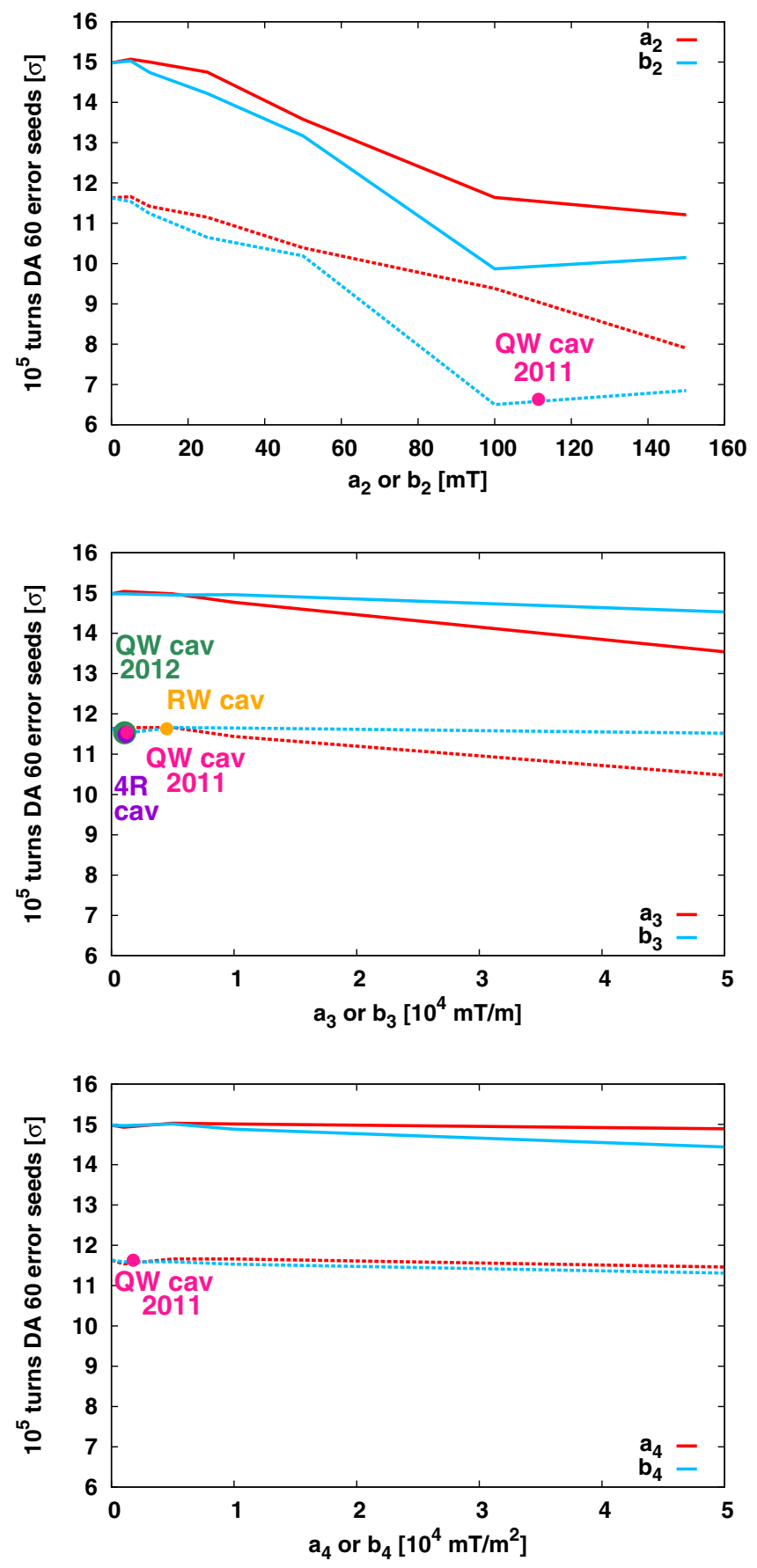

FIG. 3. Average (solid) and minimum (dashed) DA of the 60 realizations of the errors as a function of normal and skew quadrupolar (top), sextupolar (middle) and octupolar (bottom) component. In all cases the scenario simulated in $\mathrm{H}_{\mathrm{IP} 1}-\mathrm{H}_{\mathrm{IP} 5}$ the multipole value quoted in the $\mathrm{x}$-axis is per side per IP. The expected minimum DA for each CC prototype is indicated.

components have very little effect on the DA. The minimum DA is driven in all cases by the magnets nonlinearities in the lattice. These results and plots are general and are presented as a function of multipole coefficient, and the actual multipole strengths for the $\mathrm{CC}$ designs are shown as 
dots on the plots. Therefore the impact of general cavities, with differing multipole content, can be assessed.

\section{CONCLUSIONS}

In this paper we have considered the multipole content of the CC designs for the luminosity upgrade of the LHC, and the resulting impact on long term machine dynamics. After presenting three different approaches for computing the rf multipoles of a crab cavity, the rf multipoles for the different CC designs have been calculated. The agreement between the methods was excellent, and the role of symmetry in producing a negligible $b_{2}$ discussed. The multipoles were used to evaluate the long term stability of the LHC in the presence of the competing CC designs. This was done by using a dynamic aperture study in the presence of machine magnetic errors, and calculation of optical aberrations. It was shown that a symmetric cavity design is a very important requirement to suppress $b_{2}$ and not produce tuneshifts that largely degrade the dynamic aperture. This was illustrated for the QWCAV 2011 design. The dynamic aperture as a function of rf multipole coefficient was computed to illustrate the strength of the dependence and, for the symmetric cavity designs, the rf multipoles were shown to have a negligible impact on the DA in both $\mathrm{H}_{\mathrm{IP1} 1}-\mathrm{H}_{\mathrm{IP} 5}$ and $\mathrm{V}_{\mathrm{IP1}}-\mathrm{H}_{\mathrm{IP} 5}$ scenarios. The new calculations presented here of the rf multipole content and the long term stability shows the CC designs show no major impact in the dynamic aperture. At the present time attempts to measure the multipoles in the actual prototypes are ongoing.

Further studies taking into account higher order multipoles above the octupolar component should be carried out in the future, along with developments of alternative models of the crab cavity dynamics such as Taylor map representations [17] and generating functions [29,30].

\section{ACKNOWLEDGMENTS}

The authors would like to acknowledge Eric Mcintosh and Frank Schmidt for their help updating the SixTrack code. Rama Calaga and María Navarro for the fruitful discussions. Danilo Banfi who provided the scripts that improved the postprocessing of the large amount of data. Massimo Giovannozzi for proofreading the manuscript. This work has been supported by the High Luminosity Large Hadron Collider project.

\section{APPENDIX: RF MULTIPOLES SIXTRACK IMPLEMENTATION FORMULAS}

Single element time dependent $\mathrm{rf}$ multipoles have been included for the first time in SixTrack. This first stage of the implementation included only up to octupolar component in both, normal and skew components. The formulas are as follows,

\section{Normal quadrupole}

$$
\begin{aligned}
& \Delta x^{\prime}=-\frac{b_{2}}{B \rho} x \cos \left(\frac{\omega z}{c}+\phi_{s}+\phi_{\mathrm{RF}, q u a d}\right) \\
& \Delta y^{\prime}=\frac{b_{2}}{B \rho} y \cos \left(\frac{\omega z}{c}+\phi_{s}+\phi_{\mathrm{RF}, q u a d}\right) \\
& \Delta \delta=\frac{1}{2} \frac{b_{2}}{B \rho}\left(x^{2}-y^{2}\right) \sin \left(\frac{\omega z}{c}+\phi_{s}+\phi_{\mathrm{RF}, \text { quad }}\right) \frac{\omega}{c}
\end{aligned}
$$

Normal sextupole

$$
\begin{aligned}
& \Delta x^{\prime}=-\frac{b_{3}}{B \rho}\left(x^{2}-y^{2}\right) \cos \left(\frac{\omega z}{c}+\phi_{s}+\phi_{\mathrm{RF}, \text { sext }}\right) \\
& \Delta y^{\prime}=2 \frac{b_{3}}{B \rho} x y \cos \left(\frac{\omega z}{c}+\phi_{s}+\phi_{\mathrm{RF}, \text { sext }}\right) \\
& \Delta \delta=\frac{1}{3} \frac{b_{3}}{B \rho}\left(x^{3}-3 x y^{2}\right) \sin \left(\frac{\omega z}{c}+\phi_{s}+\phi_{\mathrm{RF}, \text { sext }}\right) \frac{\omega}{c}
\end{aligned}
$$

\section{Normal octupole}

$\Delta x^{\prime}=-\frac{b_{4}}{B \rho}\left(x^{3}-3 x y^{2}\right) \cos \left(\frac{\omega z}{c}+\phi_{s}+\phi_{\mathrm{RF}, \mathrm{oct}}\right)$
$\Delta y^{\prime}=\frac{b_{4}}{B \rho}\left(3 x^{2} y-y^{3}\right) \cos \left(\frac{\omega z}{c}+\phi_{s}+\phi_{\mathrm{RF}, \mathrm{oct}}\right)$
$\Delta \delta=\frac{1}{4} \frac{b_{4}}{B \rho}\left(x^{4}-6 x^{2} y^{2}+y^{4}\right) \sin \left(\frac{\omega z}{c}+\phi_{s}+\phi_{\mathrm{RF}, \mathrm{oct}}\right) \frac{\omega}{c}$

Skew quadrupole

$\Delta x^{\prime}=-\frac{b_{2}}{B \rho} y \cos \left(\frac{\omega z}{c}+\phi_{s}+\phi_{\mathrm{RF}, \text { quad }}\right)$
$\Delta y^{\prime}=-\frac{b_{2}}{B \rho} x \cos \left(\frac{\omega z}{c}+\phi_{s}+\phi_{\mathrm{RF}, \text { quad }}\right)$
$\Delta \delta=\frac{b_{2}}{B \rho} x y \sin \left(\frac{\omega z}{c}+\phi_{s}+\phi_{\mathrm{RF}, \text { quad }}\right) \frac{\omega}{c}$

\section{Skew sextupole}

$$
\begin{aligned}
& \Delta x^{\prime}=-2 \frac{b_{3}}{B \rho} x y \cos \left(\frac{\omega z}{c}+\phi_{s}+\phi_{\mathrm{RF}, \text { sext }}\right) \\
& \Delta y^{\prime}=\frac{b_{3}}{B \rho}\left(y^{2}-x^{2}\right) \cos \left(\frac{\omega z}{c}+\phi_{s}+\phi_{\mathrm{RF}, \text { sext }}\right) \\
& \Delta \delta=-\frac{1}{3} \frac{b_{3}}{B \rho}\left(y^{3}-3 y x^{2}\right) \sin \left(\frac{\omega z}{c}+\phi_{s}+\phi_{\mathrm{RF}, \mathrm{sext}}\right) \frac{\omega}{c}
\end{aligned}
$$




\section{Skew octupole}

$\Delta x^{\prime}=-\frac{b_{4}}{B \rho}\left(y^{3}+3 x^{2} y\right) \cos \left(\frac{\omega z}{c}+\phi_{s}+\phi_{\mathrm{RF}, \mathrm{oct}}\right)$

$\Delta y^{\prime}=-\frac{b_{4}}{B \rho}\left(3 y^{2} x-x^{3}\right) \cos \left(\frac{\omega z}{c}+\phi_{s}+\phi_{\mathrm{RF}, \mathrm{oct}}\right)$

$\Delta \delta=\frac{b_{4}}{B \rho}\left(x^{3} y-y^{3} x\right) \sin \left(\frac{\omega z}{c}+\phi_{s}+\phi_{\mathrm{RF}, \mathrm{oct}}\right) \frac{\omega}{c}$

[1] O. S. Brunning, in 2nd HL-LHC General Meeting 2012, Frascati, Italy (2012), https://indico.cern.ch/event/183635/ contributions/318491/attachments/250937/350854/ HL-LHC-PLC.pdf.

[2] L. Rossi, Advanced Series on Directions in High Energy Physics (World Scientific, Hackensack, 2015), p. 393; L. Rossi, LHC upgrade plans: options and strategy, in Proceedings of 2nd International Particle Accelerator Conference, San Sebastián, Spain, 2011 (JACoW, San Sebastián, 2011), p. 908.

[3] S. Fartoukh, Achromatic telescopic squeezing scheme and application to the LHC and its luminosity upgrade, Phys. Rev. Accel. Beams 16, 111002 (2013).

[4] R. B. Palmer, Report No. SLAC-PUB-4707, December 1988.

[5] J. Wei, in New Directions for High-Energy Physics (Snowmass, Colorado, 1996).

[6] J. Barranco García, E. Marín Lacoma, and R. Tomás García, Luminosity studies in a traveling waist regime in the compact linear collider, Phys. Rev. Accel. Beams 16, 041001 (2013).

[7] S. Fartoukh and R. De Maria, CERN Technical Report No. CERN-ATS-2012-136, 2012.

[8] R. Calaga, Crab cavities for the LHC upgrade, in Chamonix 2012 Workshop on LHC performance, CERN Reports No. CERN-ATS-2012-069 (2012) edited by C. Carli, https://cds.cern.ch/record/1424362?ln=en, p. 363.

[9] R. De Maria and S. Fartoukh, Report No. CERN-sLHCPROJECT-Report-0055, 2011.

[10] J. R. Delayen et al., in 5th LHC Crab Cavity workshop, Geneva, Switzerland, 2011 (CERN, Geneva, 2011).

[11] G. Burt et al., in 5th LHC Crab Cavity workshop, Geneva, Switzerland, 2011 (CERN, Geneva, 2011).

[12] I. Ben-Zvi et al., in 5th LHC Crab Cavity workshop, Geneva, Switzerland, 2011 (CERN, Geneva, 2011).

[13] M. Navarro Tapia, A. Grudiev, and R. Calaga, RF multipolar characterization of the latest LHC deflecting cavities, in Proceedings of the 4th International Particle
Accelerator Conference, Shangai, China, 2013 (JACoW, CERN, Shanghai, 2013), p. 2402.

[14] K. Ohmi et al., Response of colliding beam-beam system to harmonic excitation due to crab-cavity rf phase modulation, Phys. Rev. Accel. Beams 14, 111003 (2011).

[15] Y.-P. Sun, R. Assmann, R. Tomás, and F. Zimmermann, Crab dispersion and its impact on the CERN Large Hadron Collider collimation, Phys. Rev. Accel. Beams 13, 031001 (2010).

[16] Y.-P. Sun, R. Assmann, J. Barranco, R. Tomás, T. Weiler, F. Zimmermann, R. Calaga, and A. Morita, Beam dynamics aspects of crab cavities in the CERN Large Hadron Collider, Phys. Rev. Accel. Beams 12, 101002 (2009).

[17] D. R. Brett, R. B. Appleby, R. De Maria, J. Barranco Garcia, R. Tomás Garcia, B. Hall, and G. Burt, Accurate crab cavity modeling for the High Luminosity Large Hadron Collider, Phys. Rev. Accel. Beams 17, 104001 (2014).

[18] D. A. Goldberg and G. R. Lambertson, Dynamic devices. A primer on pickups and kickers, AIP Conf. Proc. 249, 537 (1992).

[19] Ansys HFSS, http://www.ansys.com.

[20] W. Panofsky and W. Wenzel, Some considerations concerning the transverse deflection of charged particles in radiofrequency fields, Rev. Sci. Instrum. 27, 967 (1956).

[21] D. T. Abell, Numerical computation of high-order transfer maps for rf cavities, Phys. Rev. Accel. Beams 9, 052001 (2006).

[22] D. R. Brett, R. B. Appleby, G. Burt, and B. Hall, Particle trajectories in a four rod crab cavity, Nucl. Instrum. Methods Phys. Res., Sect. A 734, 79 (2014).

[23] P. Hagen, CERN Technical Report No. LHC-PROJECTReport-1056, 2008.

[24] W. Herr, CERN Technical Report No. CERN-LHC-Note258, 1993.

[25] J. Barranco et al., in 4th Joint HiLumi LHC-LARP Annual Meeting (KEK, Tsukuba, Japan, 2014).

[26] J. Barranco García et al., CERN Technical Report No CERN-ATS-2012-120, 2012.

[27] E. McIntosh and R. De Maria, CERN, Report No. CERNATS-Note-2012-089 TECH, 2012.

[28] Y. Nosochkov et al., CERN Technical Report CERNACC2013-0132, 2013.

[29] A. Wolski, J. Gratus, and R. Tucker, Symplectic integrator for s-dependent static magnetic fields based on mixedvariable generating functions, J. Instrum. 7, P04013 (2012).

[30] R. B. Appleby and D. T. Abell, Accurate dynamics in an azimuthally-symmetric accelerating cavity, J. Instrum. 10, P02005 (2015). 